\title{
Effectiveness Analysis of Rolling Bearing Fault Detectors Based On Self-Organising Kohonen Neural Network - A Case Study of PMSM Drive
}

Research article

Kamila Jankowska, Pawel Ewert*

Department of Electrical Machines, Drives and Measurements, Wroclaw University of Science and Technology, Wybrzeze Wyspianskiego 27, 50-370 Wroclaw, Poland

Received: May 14, 2021; Accepted: July 01, 2021

\begin{abstract}
Due to their many advantages, permanent magnet synchronous motors (PMSMs) are increasingly used in not only industrial drive systems but also electric and hybrid vehicle drives, aviation and other applications. Unfortunately, PMSMs are not free from damage that occurs during their operation. It is assumed that about $40 \%$ of the damage that occurs is related to rolling bearing damage. This article focuses on the use of Kohonen neural network (KNN) for rolling bearing damage detection in a PMSM drive system. The symptoms from the fast Fourier transform (FFT) and Envelope (ENV) Analysis of the mechanical vibration acceleration signal were analysed. The signal ENV was obtained by applying the Hilbert transform (HT). Two neural network functions are discussed: a detector and a classifier. The detector detected the damage and the classifier determined the type of damage to the rolling bearing (undamaged bearing, damaged rolling element, outer or inner race). The effectiveness of the analysed networks from the point of view of the applied signal processing method, map size, type of neighbourhood radius, distance function and the influence of input data normalisation are presented. The results are presented in the form of a confusion matrix, together with $2 \mathrm{D}$ and $3 \mathrm{D}$ maps of active neurons.
\end{abstract}

Keywords: PMSM • rolling bearings • electric drive diagnostics • self-organising maps • shallow neural network

\section{Introduction}

Permanent magnets synchronous motors (PMSMs) are currently replacing induction motors (IM) in a broad range of industrial applications due to their many advantages. They are distinguished by high efficiency, high speed, high torque-to-inertia and current ratio (Jaganathan et al., 2011). But a very good parameter of motor does not mean a failure cannot happen. The failures most commonly affect the rolling bearings, as presented in this paper. They account for about $40 \%$ of all failures (He et al., 2014). The factors causing different damages may include too high current flow, high operating temperature, inadequate lubrication or corrosion. Rolling bearings are responsible for persisting constant air gaps and minimising mechanical losses.

Interrupting the industrial processes caused by unexpected failures of the motor might be very problematic. It may cause financial and other losses. For this reason, preventive maintenance strategies are necessary. Early diagnostics help to recognise and fix the problems with low cost and time (Germen et al., 2014).

Nowadays, diagnostics of electric drives are extensively developed. Most of the methods are based on the analysis of the diagnostic signal (Henao et al., 2014). In the case of bearings damage, the diagnostic signal may be, for example, noise (Lu et al., 2018), vibrations (Nkuna, 2006), current (Picot et al., 2014), and stray flux (Frosini et al., 2015). Most works are focused on using current and vibrations or both as diagnostic signal 
(Faiz et al., 2017; Immovilli et al., 2010). Based on them, it can be concluded that it is very difficult to retrieve bearing fault signatures in the current. Current-based diagnosis only detects faults characterised by a relatively low-mechanical-frequency rate. Techniques based on vibrations usually used in the industry allow detection of even incipient faults. Subsequently, measurements are processed using one or more of the signal analysis methods. Methods used for signal processing may be fast Fourier transform (FFT) (Rosero et al., 2009), Hilbert transform (HT) (Skora et al., 2019) and Variational Mode Decomposition (VMD) (Liu et al., 2019). The use of any of the abovementioned methods requires expert evaluation, which may turn out to be the weakest link of the diagnostic system. The use of neural networks (NNs) in the diagnostic process can improve the process and make it fully automated (Ewert et al., 2020).

Most research works concerning the electric drive diagnostic process describe the usage of deep neural networks (DNNs), which are gaining more popularity due to the development of deep learning methods. See for example Ullah et al. (2020). The authors presented the diagnostics of rolling bearings of the PMSM drive using a convolutional neural network (CNN) with the use of stator current and vibration measurements. Parallel processing of stator current frequency spectrum and raw vibration signal allowed the efficiency to improve to $96 \%$ in relation to diagnostic based on one signal.

Currently, classic shallow NNs are overlooked in research works due to the growing interest of scientists in DNNs in PMSM drive diagnostics. It needs to be highlighted that they have several important advantages that should be taken into account. One of the most important is much simpler architecture, significantly shorter learning process and lower computational complexity, still preserving high efficiency.

The article by Akar et al. (2015) can be cited as an example where NNs are used to detect eccentricity and damage to rolling bearings in the PMSM, especially the multilayer perceptron (MLP). The effectiveness was tested for variable speed and load torque values. Under the conditions of the highest load and speed, $100 \%$ efficiency was achieved. The stator current measurement was used as a diagnostic signal.

Self-organising maps (SOMs), also known as Kohonen neural network (KNN), are less frequently used as damage classifiers of mechanical faults. In the literature, we can find examples of using SOM for estimation of optimal parameters of motor (Jaganathan et al., 2011), damage classifying of electrical faults (Skowron et al., 2019), or detection and classification of rolling bearing damage (Ewert et al., 2021).

An example of using SOM to detect damage to rolling bearing in PMSM is presented in the literature (Nkuna, 2006). Neural network was responsible for classifying the fault level. For diagnostic signal, vibration measurement was used. FFT and wavelet transform methods were used to process the signal. The research was conducted for five different loading conditions, five different shaft speeds, and eight bearing failures. The proposed SOM made it possible to detect a damaged bearing with a low topological error of about \pm 0.04 but a moderate quantisation error of \pm 0.5 .

Zhang et al. (2020) proposed a different approach using signal processing based on VMD and then the selforganising feature map for rolling bearing fault diagnosis. This allows the detection of faults during nonstationary work operations. The collected data contains four types of motor conditions: an outer race fault, an inner race fault, a rolling element fault, and normal operation. Results of fault classification are presented on a 2D map. The map shows active areas for different types of faults. The proposed method allows an accuracy of about $87 \%$ to be obtained.

In this article, the effectiveness analysis of neural detectors of damage to rolling bearings in PMSM based on KNN is presented. Tested networks differ from other shallow NNs by the ability to detect hidden relationships between input data, which is their special advantage. Another advantage of KNN is their simple structure. In addition, it was decided to combine FFT with envelope (ENV), which improves the effectiveness of detection, as shown in the work of Skora et al. (2019). As mechanical vibrations are commonly used diagnostic signals in industrial plants, the article proposes a neural detector based on this signal. This article contains six parts. In the first part, a review of the literature on the diagnosis of rolling bearings, with particular emphasis on NNs and the motivation of the research, is presented. In the second section, the KNNs are shortly characterised. In the third and fourth sections, experimental set-up and the process of designing neural detectors are described. The results of testing rolling bearing fault classifiers based on KNNs are presented in the fifth section. Effectiveness was analysed for various map sizes, distance functions and neighbourhood radius. Vibration measurement was chosen as a diagnostic signal and processed by HT and FFT. The last part of this paper contains a short summary. 


\section{Principles of KNNs}

SOMs are feedforward NNs, without hidden layers, which simplify the network's architecture. The network is built with input and output layers. Each input layer component is linked to every output layer component. The output signal $y_{k}$ of the $k$ th neuron is described by the following equation:

$$
y_{k}=\mathbf{W}^{T} \mathbf{x}=\|\mathbf{W}\|\|\mathbf{x}\| \cos \mathrm{f}_{k}
$$

In SOM, the input $\boldsymbol{x}$ and weights $\boldsymbol{W}$ vectors are normalised, while the output value of $y_{k}$ (neuron activation) is determined by the angular difference $\phi_{k}$ between the vectors $\boldsymbol{x}$ and $\boldsymbol{W}$. In Figure 1, the general structure of KNN is presented.

SOMs distinguish the learning process from other shallow NNs. The competition and neighbourhood mechanisms are the basis of the network learning process. The learning process is unsupervised. Due to the unsupervised learning process, SOM recognises unknown relationships between input data. Initially, neurons are placed randomly. The weights assigned to neurons are in the range of $\langle 0.1>$. In subsequent iterations, individual neurons respond to input vectors in varying degrees. The competition is that the neuron that reacts most strongly wins. Winner takes all (WTA) and winner takes most (WTM) are the two main types of learning algorithms. The most important part of both algorithms is the adaption process. It is an element that differs from WTA and WTM. Adaptation is bringing the winning neuron closer to the input pattern. In the WTA method, only the winning neuron is subject to adaptation. It is different in the WTM algorithm, where neurons from an appropriately defined neighbourhood (e.g. Gauss neighbourhood function) also can be activated (weight adaptation). To determine how much a neuron and its neighbour's weight vectors are updated when new patterns are presented, the learning rate $\alpha$ is used (Breard, 2017).

Network performance is usually presented as 2D maps that represent the distribution of neurons activating for a particular data input. Kohonen's map can be built on the basis of various types of grids: rectangular and hexagonal. Neurons can also be placed randomly. The map size can be arbitrary. Multidimensional maps are also allowed.

Map topology, with reference to which the spatial relation between data points can be determined, is in most cases judged by quantisation error $\left(Q_{e r r}\right)$ and topographic error $\left(E_{T}\right) . Q_{e r r}$ is the basic quality measure for evaluating

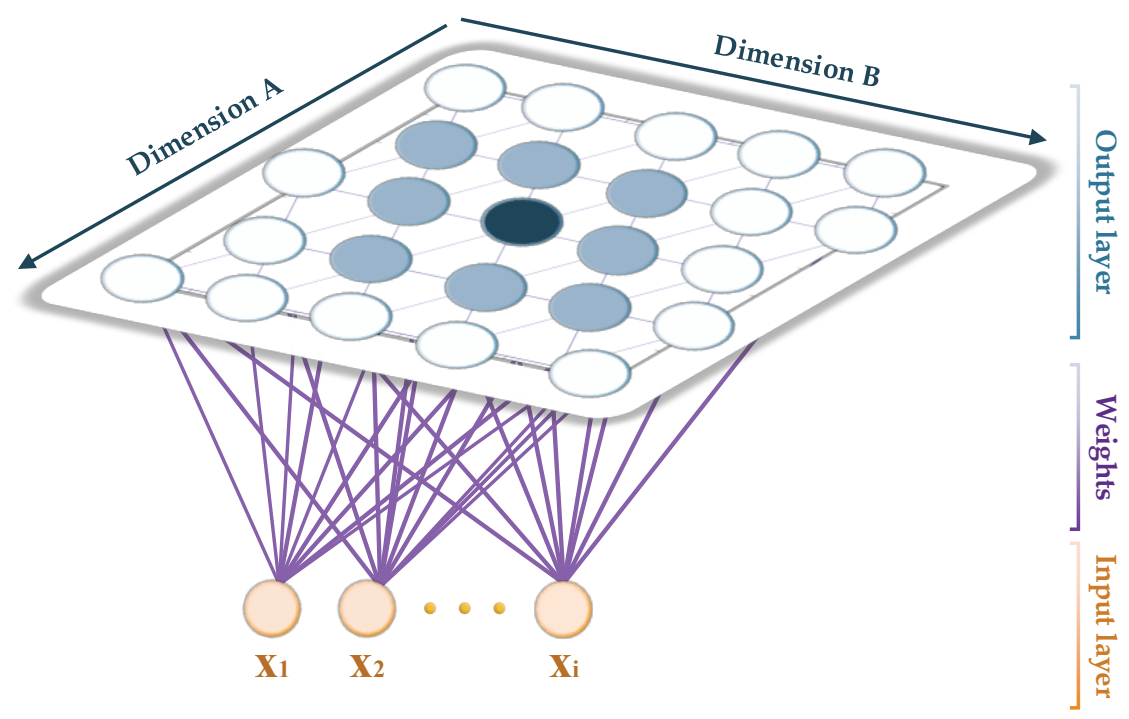

Fig. 1. General architecture of KNN. KNN, Kohonen neural network. 
SOMs (Kohonen, 2001). $Q_{\text {err }}$ is an average distance between the data points and the map nodes to which they are mapped $(M)$, with smaller values indicating a better fit. The value for a map is calculated using the following formula-

$$
Q_{e r r}(M)=\frac{1}{n} \sum_{i=1}^{n}\left\|\mathrm{f}\left(x_{i}\right)-x_{i}\right\|
$$

where $n$ is the number of data points in the training data and $\phi$ is the mapping from the input space to the SOM $M$ (Breard, 2017).

Topographic error is a measure of how well map structure is organised. The aim is for the topographic error to be 0 . This ensures that every two neurons whose weight vector is closest to the input vector are also the closest neighbours on the map. Topographic error is expressed by the following form:

$$
T_{E}(M)=\frac{1}{n} \sum_{i=1}^{n} t\left(x_{i}\right)
$$

In Eq. (3), $t\left(x_{i}\right)$ takes the value 1 or 0 . When two closest neurons for the vector input are connected with each other and they are the closest neighbours in the map space $t\left(x_{i}\right)=1$; otherwise $t\left(x_{i}\right)=0$. This measure assesses how well individual data points are mapped to the nodes (Breard, 2017).

\section{Laboratory Set-up}

The neural classification preparation cycle begins with collecting data from a real object or simulation. As a diagnostic signal for fault detectors, vibration measurement was selected. The research was carried out on PMSM (Lenze MCS14H15), with a rated power of $2.5 \mathrm{~kW}\left(U_{N}=325 \mathrm{~V}, I_{N}=6.6 \mathrm{~A}, T_{N}=21 \mathrm{~N} \cdot \mathrm{m}, f_{s}=100 \mathrm{~Hz}, n_{N}=1,500 \mathrm{r} / \mathrm{min}\right.$, $\left.p_{p}=4\right)$. Laboratory set-up also includes Lenze E84VTCE40245X0 converter to power and control tested motor. To generate load torque, a second Lenze motor was used, powered by the same type of converter. Both motors and load inverter are shown in Figure 2. The measurements were taken through the NI PXle-4492 measurement card and the NI PXle-1082 computer. In the experiments carried out as part of the research, bearings with modelled damage to the outer and inner raceways and rolling elements, and bearings without a defect, were used to carry out vibration measurements using the DeltaTron 4506 accelerometer from Brüel and Kjær. The applied triaxial piezoelectric accelerometer was characterised by a frequency range of $0.3-5.5 \mathrm{kHz}$ in the $x-a x i s$ and $0.6-3 \mathrm{kHz}$ in the $y$ and z-axes, respectively. The sampling rate of the measurement card is set to $16.384 \mathrm{kHz}$. To extract characteristic symptoms, spectra were analysed in the low-frequency range up to $500 \mathrm{~Hz}$.
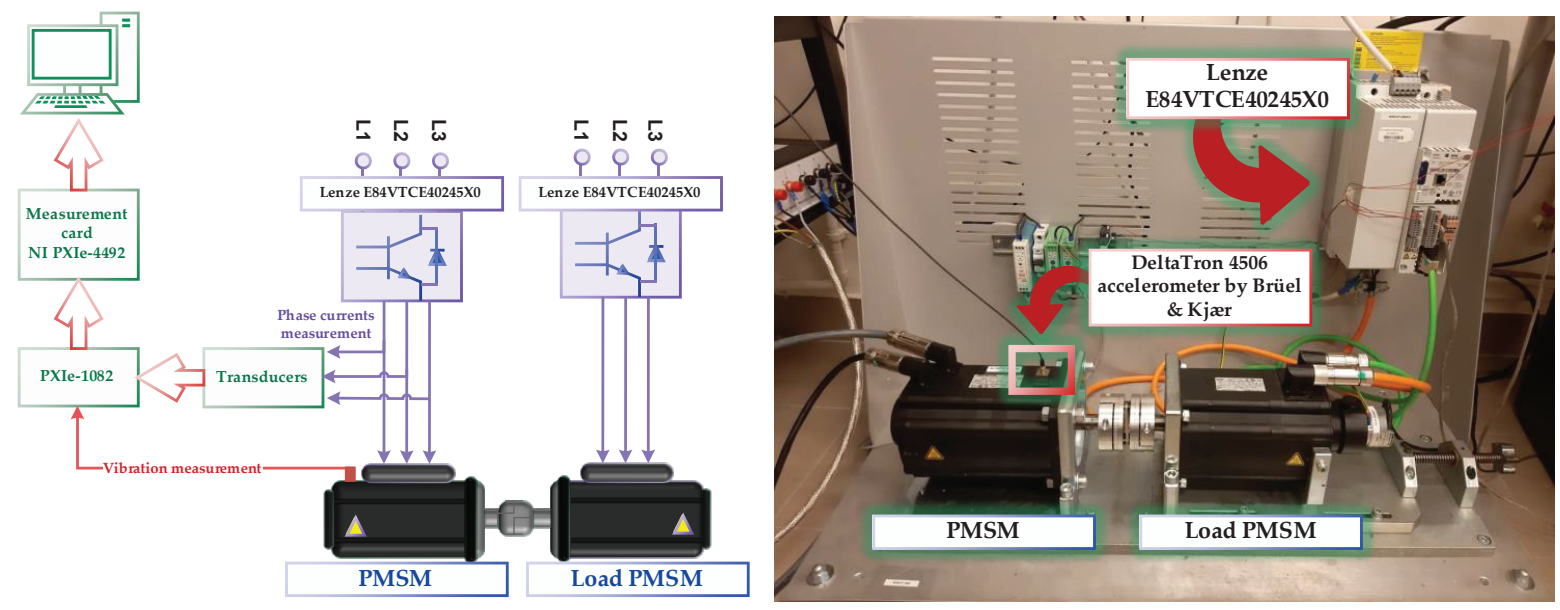

Fig. 2. Schematic diagram and photo of the laboratory stand. PMSM, permanent magnets synchronous motors. 
During the research, the vibration acceleration measured was carried out in three axes. Then measurement results were analysed. Preliminary analysis of the data obtained from each axis of measured mechanical vibrations allowed choosing the one that responds most (large amplitude of characteristic symptoms, uniform change of amplitude under the influence of load and supply frequency changes) to the damage of the bearing structural element. The greatest response to bearing damage was visible in the signal on the $x$-axis - measurement of vibrations was carried out in the radial direction; so, only this signal was used for further analysis. In order to obtain an effective learning process and the ability to verify the effectiveness of the network, measurements were carried out in two series with variable supplying voltage frequency and variable load torque.

\section{Designing Process of Fault Detectors Based on KNNs}

After the measurement of the selected diagnostic signal, the data for the network inputs must be appropriately processed. For this purpose, signal analysis methods are used. Then prepared data should be divided into training and testing vectors. The quantity of learning patterns is very important. Too few may cause mistakes with faults classification; too many are associated with a long computation time. The vector of learning patterns must be sufficiently complete. The full process of designing neural detectors is presented in Figure 3.

As signal processing methods, FFT and ENV were used. The use of the Fourier transform allowed determination of the amplitudes of the characteristic failure frequencies using an application developed in the Labview environment. The FFT analysis used a flat top window and the results were presented on a linear scale. Signals measured for $10 \mathrm{~s}$ were analysed so that the obtained spectra had a resolution of $0.1 \mathrm{~Hz}$. Comparison of sample spectra for a healthy bearing and a bearing with a modelled inner and outer race damage is shown in Figure 4. The learning and testing of KNN were carried out in Matlab environment, where we can modify the following arguments: map size, number of epochs, neighbourhood radius, type of grid, and neighbourhood function. During the learning process, the number of epochs was set to a constant value of 500 . The network parameterisation process started with the default values: map size $8 \times 8$, neighbourhood radius 3 , hextop mesh type, and linkdist neighbourhood function. Then, the influence of individual parameters was investigated, with the constancy of the others. The most important results are presented in this article.

Frequency characteristics for inner race, $f_{i r}$, outer race, $f_{\text {or }}$ and rolling element, $f_{r e}$, were calculated as follows, also using Labview environment:

$$
\begin{aligned}
& f_{i r}=f_{r}(k \cdot 4.95 \pm l) \\
& f_{o r}=f_{r}(k \cdot 3.05 \pm l) \\
& f_{r e}=f_{r}(k \cdot 3.99 \pm l)
\end{aligned}
$$

where $k=1,2,3$ and 4 and $I=0.1$. Equations include coefficients obtained from the bearing producer.

The rotating frequency, $f_{r}$, was calculated as:

$$
f_{r}=\frac{f_{s}}{p_{p}}
$$

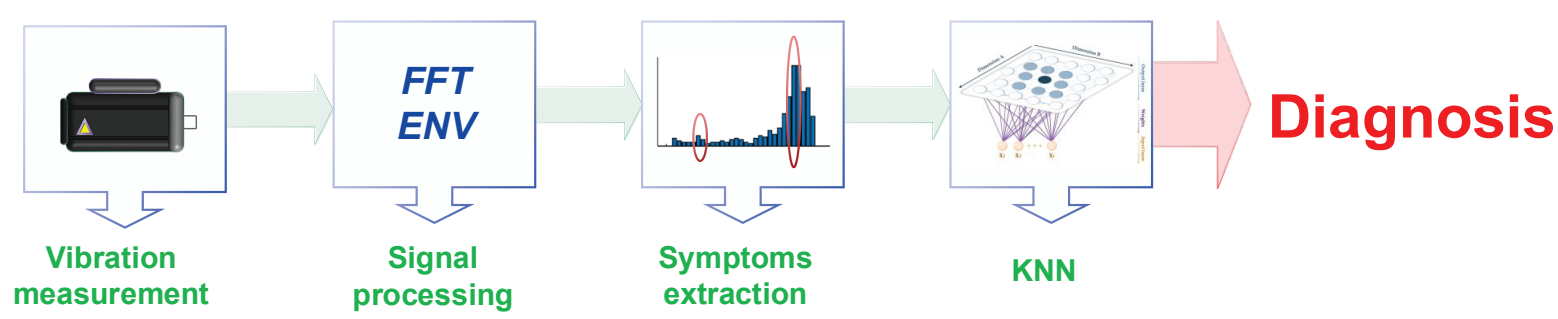

Fig. 3. Schematic of designing process of neural fault detector. ENV, envelope; FFT, fast Fourier transform; KNN, Kohonen neural network. 
a)

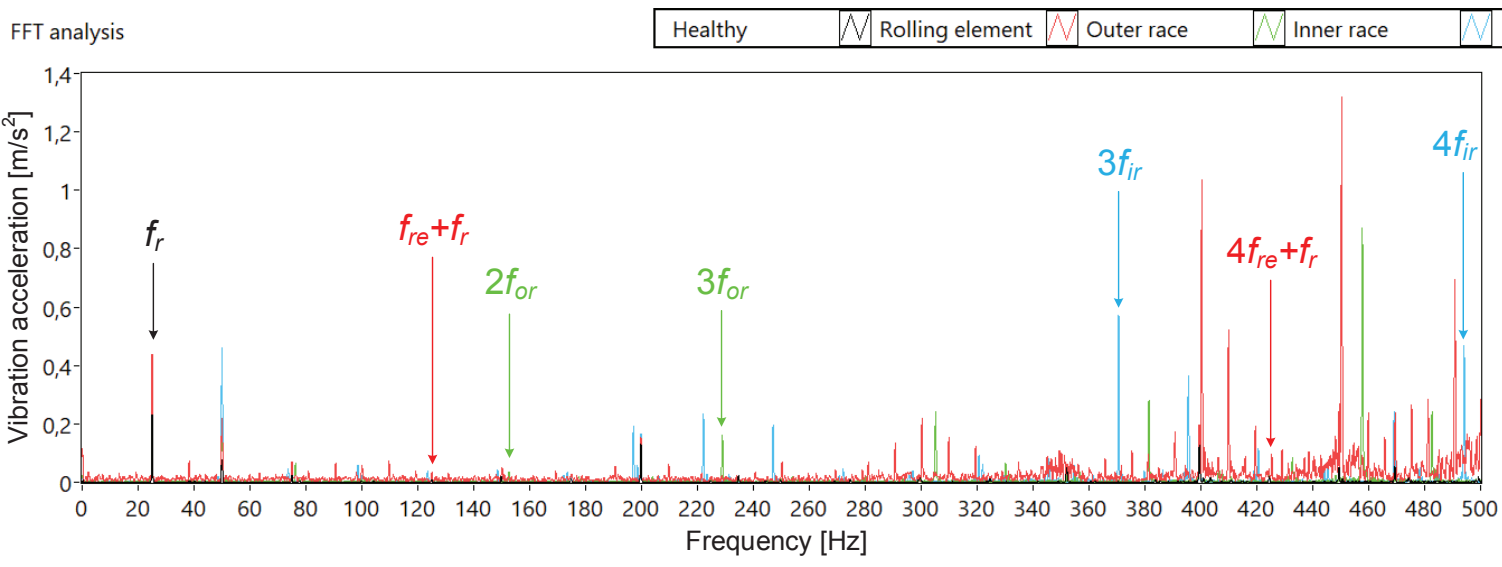

b)

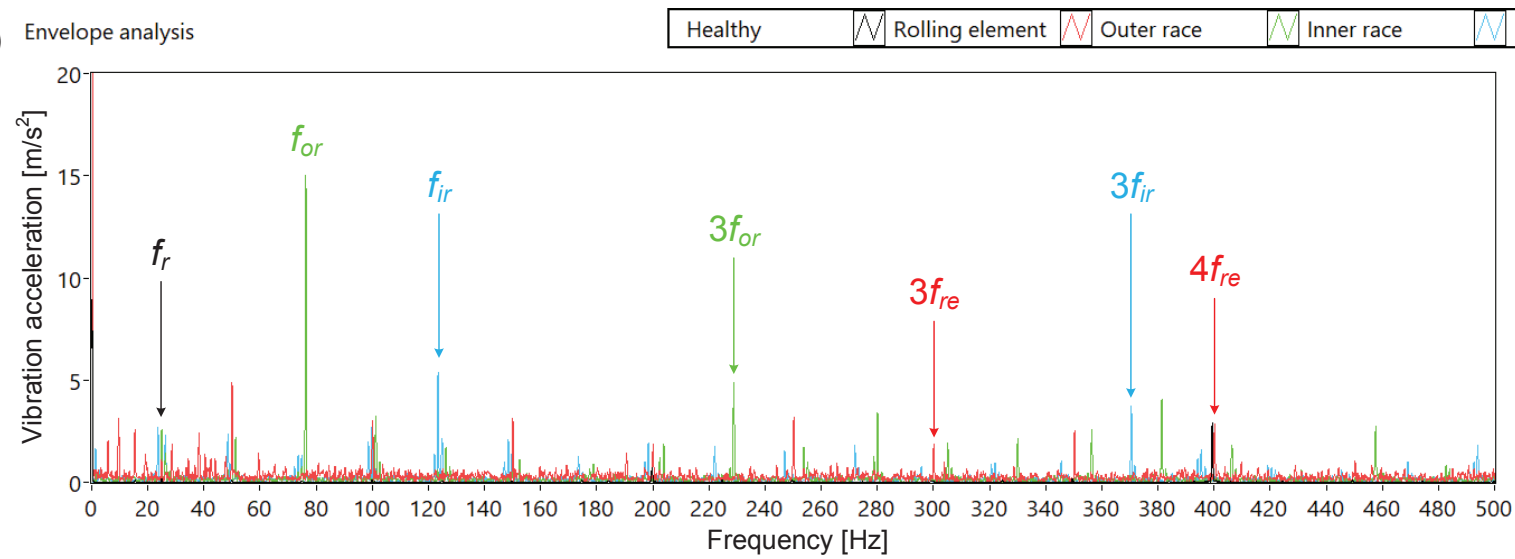

Fig. 4. Examples of vibration spectra for all types of damages under nominal load and $f_{s}=100 \mathrm{~Hz} F F T$ analysis (a), ENV analysis (b). ENV, envelope; FFT, fast Fourier transform.

Table 1. Examples of selected frequencies for FFT-based detector

\begin{tabular}{lrrrrrrr}
\hline$f_{s}[\mathrm{~Hz}]$ & $f_{r}[\mathrm{~Hz}]$ & $3 f_{i r}[\mathrm{~Hz}]$ & $4 f_{i r}[\mathrm{~Hz}]$ & $2 f_{o r}[\mathrm{~Hz}]$ & $3 f_{o r}[\mathrm{~Hz}]$ & $f_{r e}+f_{r}[\mathrm{~Hz}]$ & $4 f_{r e}+f_{r}[\mathrm{~Hz}]$ \\
\hline \hline 20 & 5 & 74.25 & 99 & 30.5 & 45.75 & 24.95 & 84.8 \\
60 & 15 & 222.75 & 297 & 91.5 & 137.25 & 74.85 & 254.4 \\
100 & 25 & 371.25 & 495 & 152.5 & 228.75 & 124.75 & 424 \\
\hline
\end{tabular}

FFT, fast Fourier transform.

Table 2. Examples of selected frequencies for ENV-based detector

\begin{tabular}{lcrrrrrc}
\hline$f_{s}[\mathrm{~Hz}]$ & $f_{r}[\mathrm{~Hz}]$ & $f_{i t}[\mathrm{~Hz}]$ & $3 f_{i t}[\mathrm{~Hz}]$ & $f_{\text {or }}[\mathrm{Hz}]$ & $3 f_{\text {or }}[\mathrm{Hz}]$ & $3 f_{r e}[\mathrm{~Hz}]$ & $4 f_{r e}[\mathrm{~Hz}]$ \\
\hline \hline 20 & 5 & 24.75 & 74.25 & 15.25 & 45.75 & 59.85 & 79.8 \\
60 & 15 & 74.25 & 222.75 & 45.75 & 137.25 & 179.55 & 239.4 \\
100 & 25 & 123.75 & 371.25 & 76.25 & 228.75 & 299.25 & 399 \\
\hline
\end{tabular}

ENV, envelope.

Then the amplitudes of the calculated frequencies were determined. The analysis covered amplitudes of 32 harmonics with frequencies associated with damage to the inner race, outer race and rolling element. Table 1 presents selected frequencies. In order to take into account the variability of the load movement and improve detection stability, the rotational frequency $f_{r}$ was chosen as an auxiliary symptom.

The second input vector was developed based on the HT analysis of the vibration acceleration signal and the next FFT of the obtained ENV. Table 2 presents selected frequencies. 
$3 f_{\text {or }}$

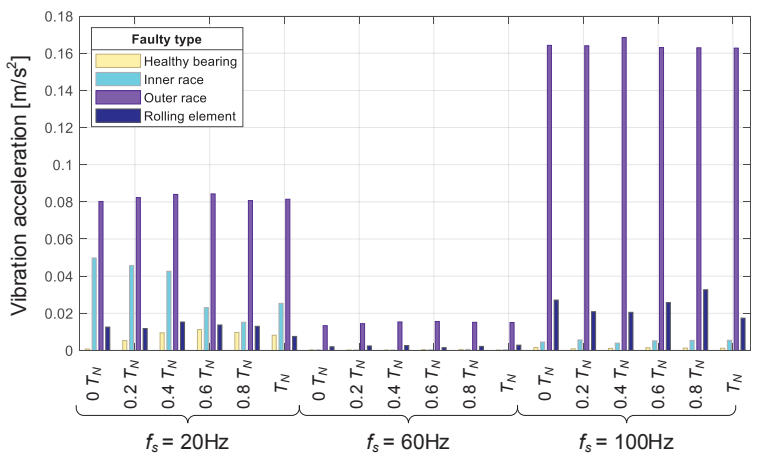

(a)

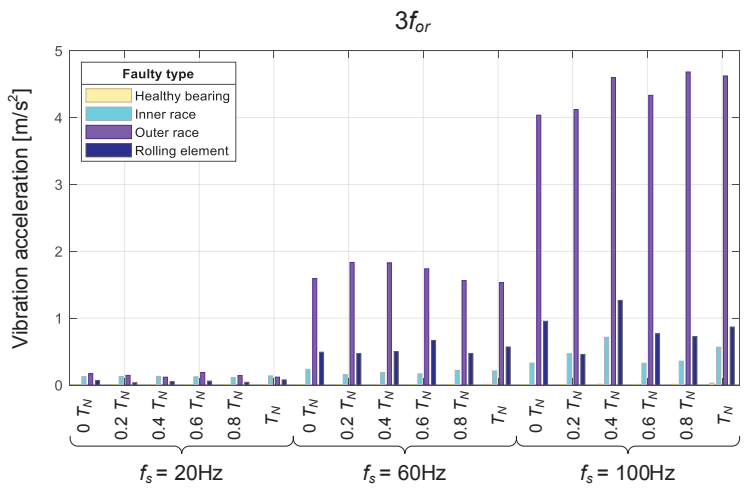

(c)

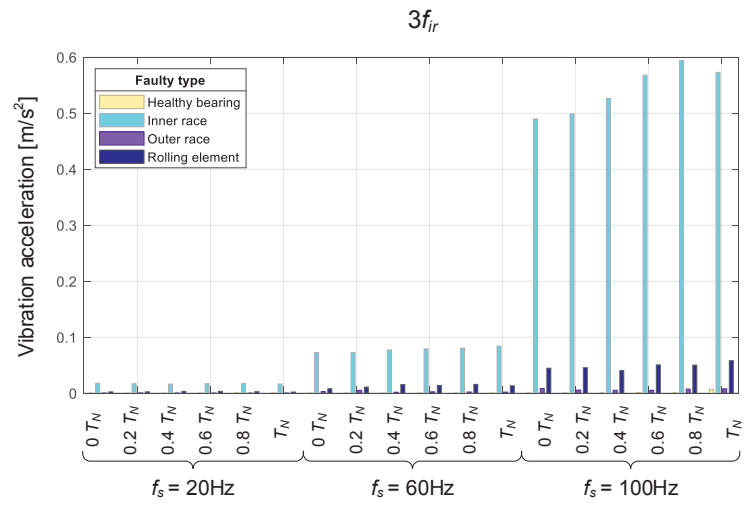

(b)

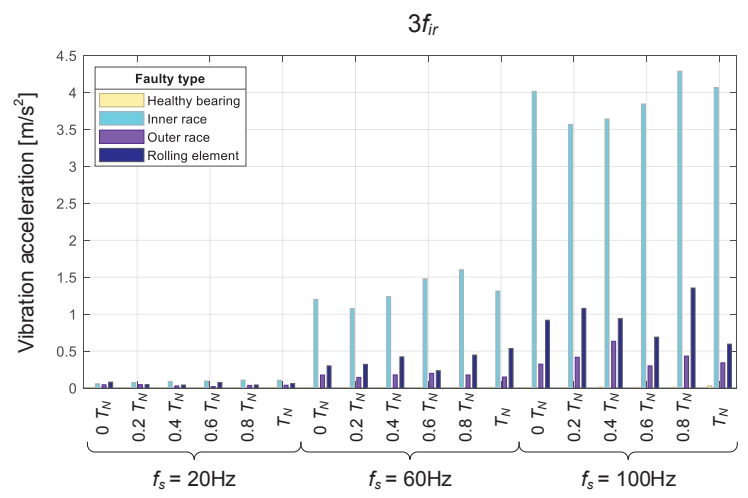

(d)

Fig. 5. Examples of harmonic frequencies amplitudes - FFT (a, b), ENV (c, d). ENV, envelope; FFT, fast Fourier transform.

After applying the HT of the signal, there was a significant increase in the amplitude values of the harmonics with frequency characteristics for individual failures. A comparison of the response to failure for several of the selected harmonics obtained for different supplying frequencies $f_{s}$ (from $20 \mathrm{~Hz}$ to $100 \mathrm{~Hz}$ ) and loads $T_{N}$ (from $0 T_{N}$ to $T_{N}$ ) between data based on ENV and only FFT is shown in Figure 5.

In the last part of inputs preparation, data was normalised to $<0.1>$ set using the following equation with coefficient $\alpha$ :

$$
x^{\prime}=\frac{x}{\sqrt{x^{2}+a}}
$$

In the research, 0.1 was taken as an optimal value for $\alpha$.

\section{Analysis of Effectiveness of Fault Detectors Based on Kohonen's Maps}

In this part, simulation results conducted in Matlab software are presented. Effectiveness was analysed for different types of distance function, values of neighbourhood radius, and the size of the map. Two types of neural detectors based on KNN were tested:

- fault detector (type I) - determining the condition of the bearing in binary way (healthy-faulty),

- fault classifier (type II) - specifying one of the bearing states (healthy, inner race fault, outer race fault, rolling element fault). KNN was tested for different map sizes $(8 \times 8,10 \times 10,16 \times 16)$, neighbourhood radius $(2,10$, 20) and distance function (linkdist, dist, mandist). 


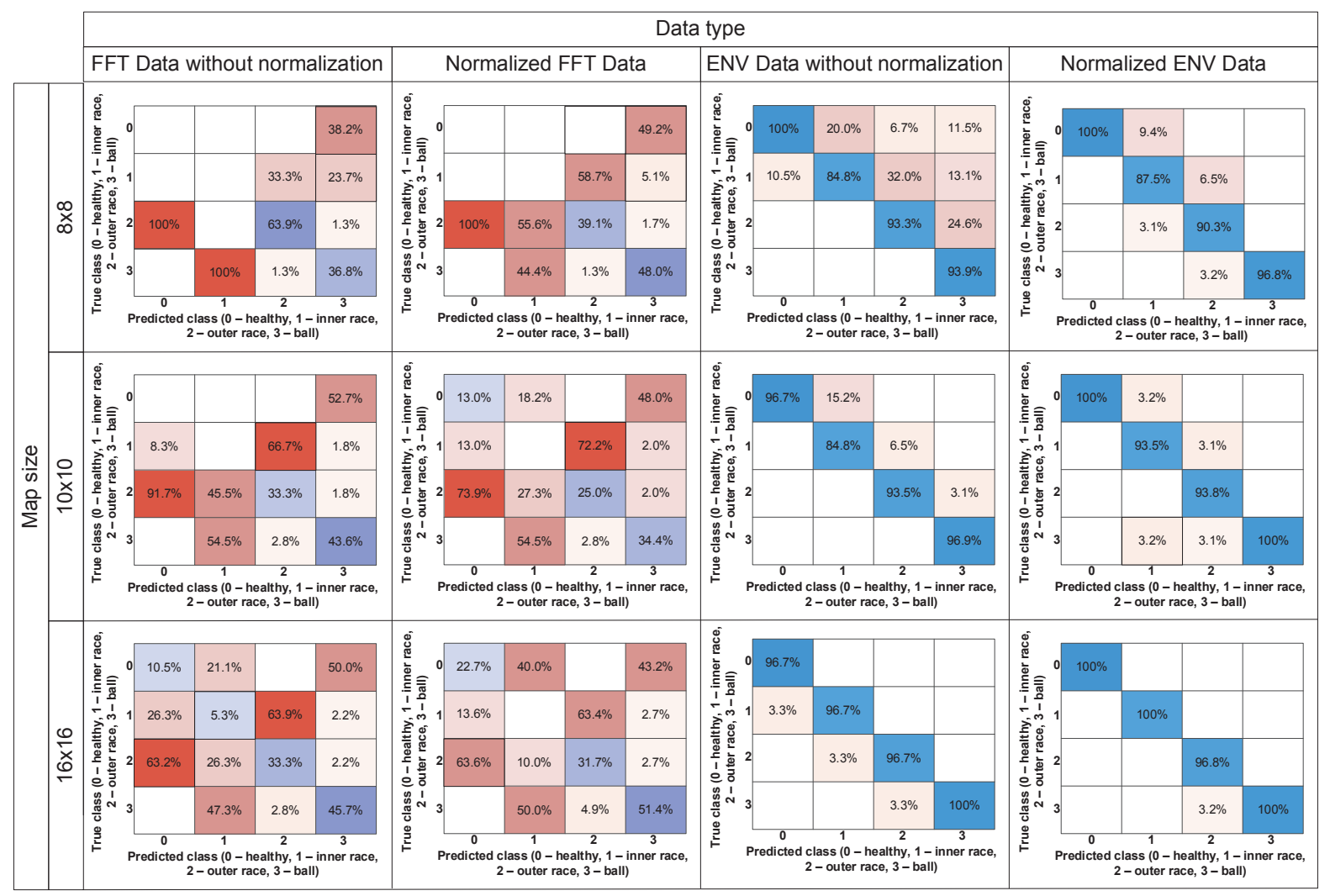

Fig. 6. Confusion matrices for different map sizes, neighbourhood radius equal to 2 and distance function dist for FFT and ENV data. ENV, envelope; FFT, fast Fourier transform.

First, results from the classifier are compared for data based on FFT and ENV with normalised data and data without normalisation to dwell on the relevance of data preparation for KNN. Confusion matrices for fault classifiers with various map sizes are submitted in Figure 6.

By comparing results, it can be observed that data based on FFT does not comply with the requirements of the diagnostic system. The percentage of errors at the level of damaged/undamaged recognition is unacceptable. Observing data without normalisation, it can be noticed that normalisation significantly improves classifier efficiency. Analysing the results of the survey, in every case lack of normalisation results case decreasing of efficiency, for those reasons other results are presented only for data based on ENV and normalised data.

Figure 7 shows the analysis for various map sizes and neighbourhood radius for fault detector. Analysing the results obtained for various types of neighbourhood radius for fault detector, it can be noticed that it has no substantial impact on KNN efficiency. Results for maps $10 \times 10$ and $16 \times 16$ are similar. Only map $8 \times 8$ shows worse results. As that initial weight of KNN is selected randomly, it cannot be determined as to which map size is better for fault detector. After every learning process, results can differ but oscillate on a similar level. Results for various distance functions are presented in Figure 8.

In the case of various distance functions, similarly to the observations recorded in the cases of various neighbourhood values, maps $10 \times 10$ and $16 \times 16$ achieve comparable efficiency. Based on map $8 \times 8$, it can be observed that dist function allows procurement of the best results. Considering the above-presented results, it can be concluded that the $10 \times 10$ map with radius equal to 2 and dist neighbourhood function is a compromise between efficiency and optimality; due to this, the map with those parameters is presented in 2D and 3D versions (active areas under faulty and healthy samples) in Figure 9.

The obtained results for fault classifier are presented in Figures 10 and 11. Fault classification does not allow procurement of such high efficiency as fault detection. As it can be seen in Figure 10, neighbourhood radius does not have a significant impact in contrast to map size, and the best results for $8 \times 8$ and $10 \times 10$ sizes were obtained for a value equal to 2 . For map $16 \times 16$, every presented value has a similar impact on detection. Grid size $16 \times 16$ allows procurement of the highest efficiency. 


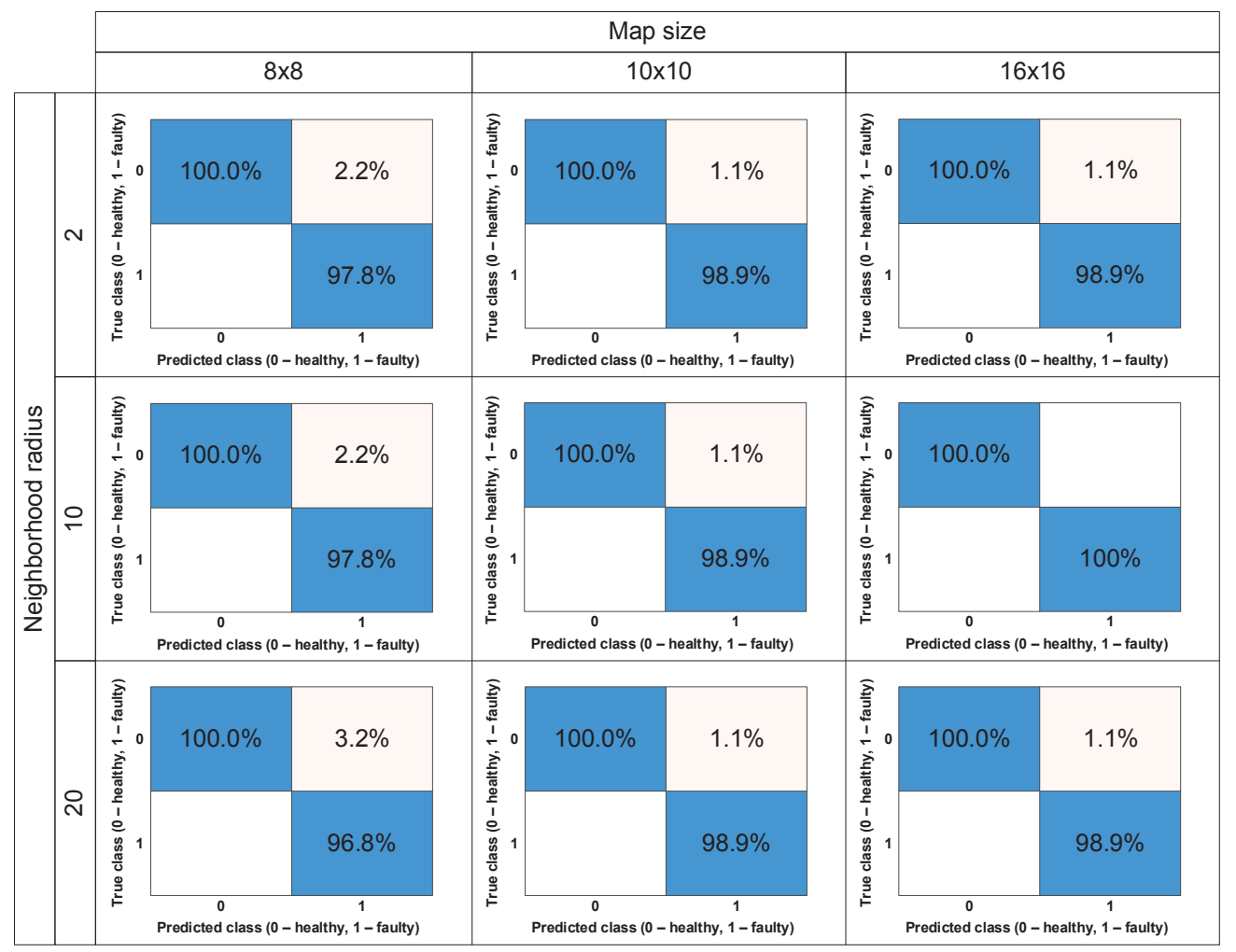

Fig. 7. Confusion matrixes for various neighbourhood radius and map sizes with distance function dist for fault detector.

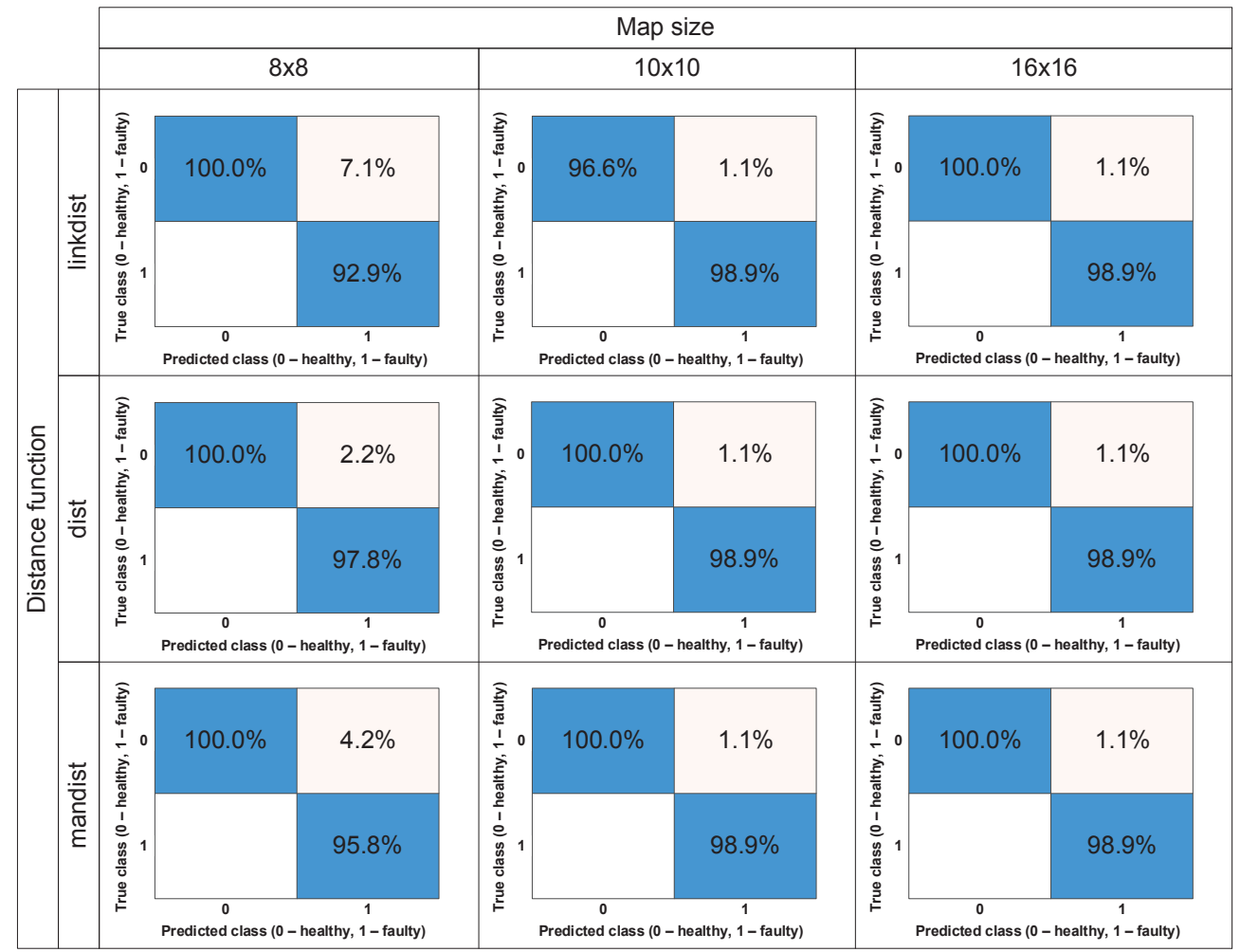

Fig. 8. Confusion matrixes for various distance functions and map sizes with neighbourhood radius equal to 2 for fault detector. 


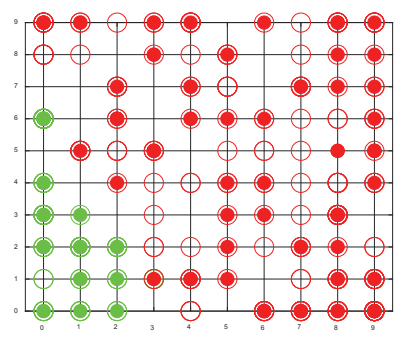

(a)

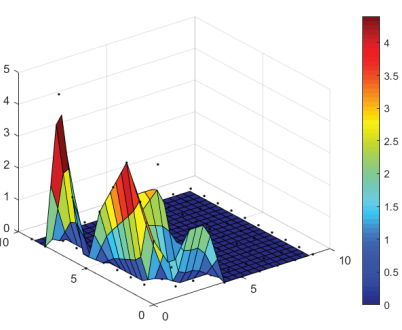

(b)

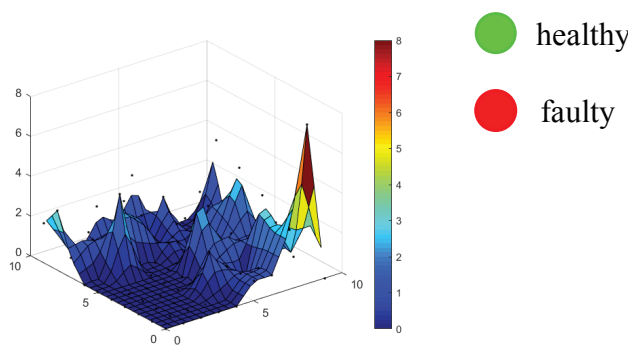

(c)

Fig. 9. 2D map - neurons activated under faulty and healthy conditions (a), active areas under healthy bearing (b), faulty bearing (c).

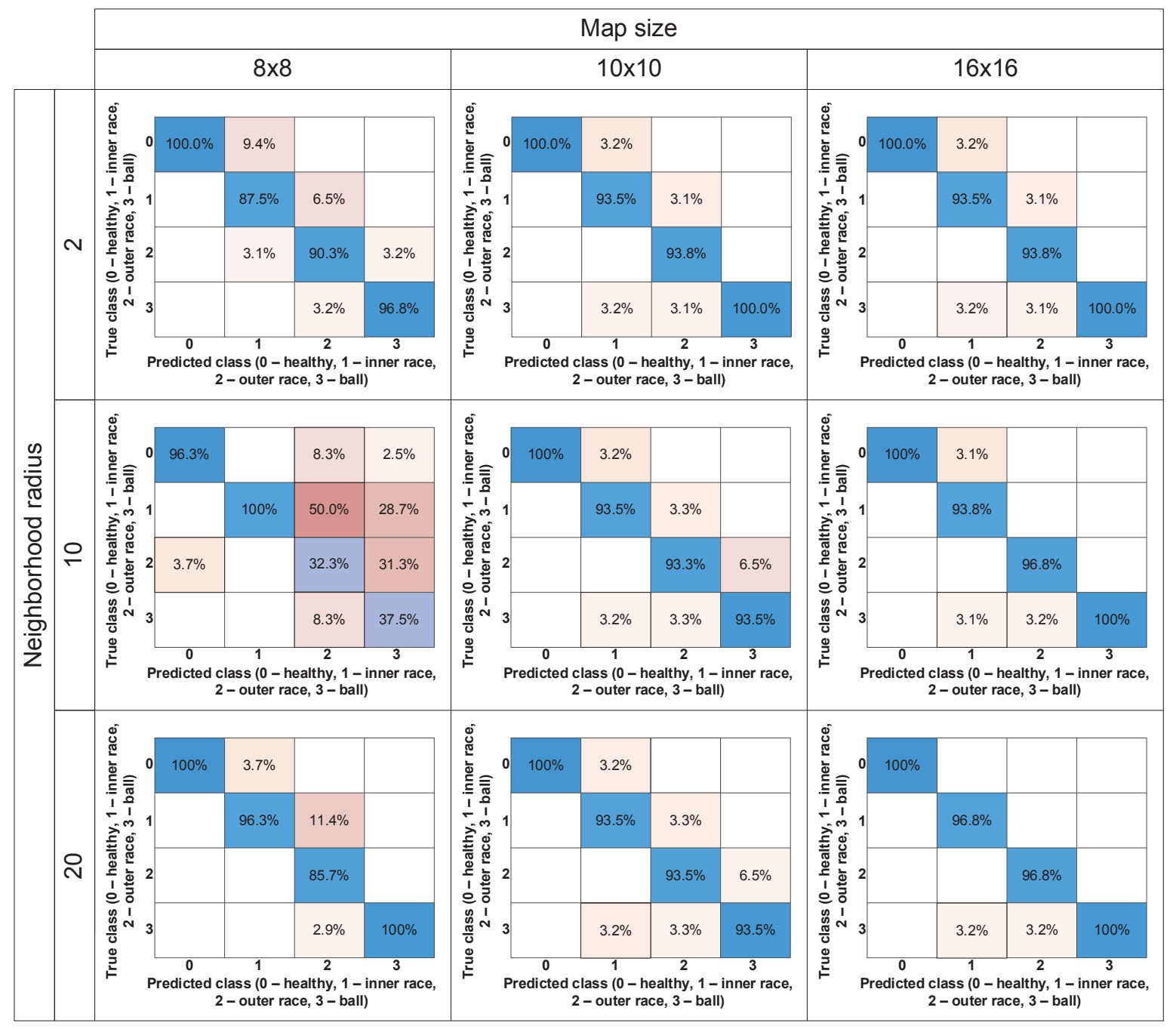

Fig. 10. Confusion matrixes for various neighbourhood radius and map sizes with distance function dist for fault classifier.

Figure 11 shows that for almost every presented map size, the distance function dist allows procurement of the best results. Most mistakes occur during the detection of inner and outer race faults. The most important element is that for every case for map, $10 \times 10$ or $16 \times 16$ distinction healthy/faulty bearing is carried out correctly. Map $16 \times 16$ with dist function and radius equal to 2 was chosen as the best solution and presented in $2 \mathrm{D}$ and $3 \mathrm{D}$ versions in Figure 12. 


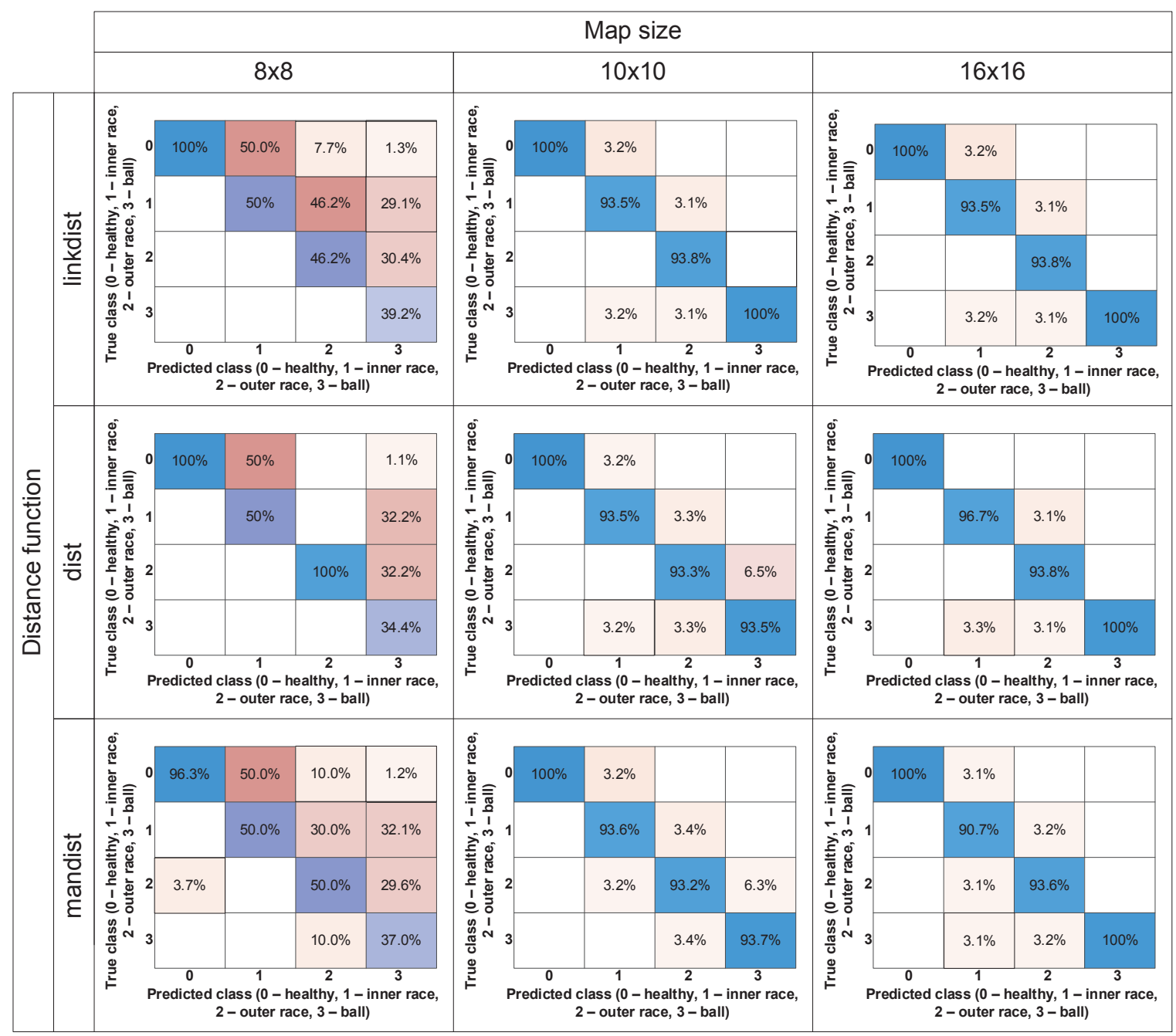

Fig. 11. Confusion matrixes for various distance functions and map sizes with neighbourhood radius equal to 2 for fault classifier.

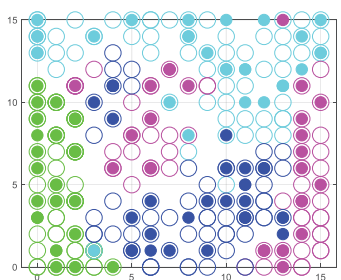

(a)

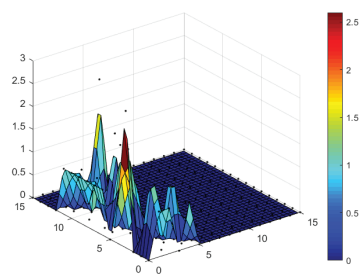

(b)

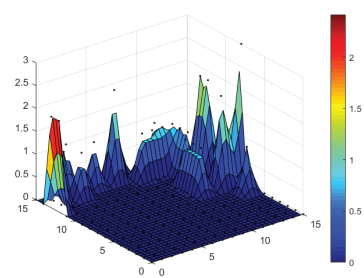

(c)

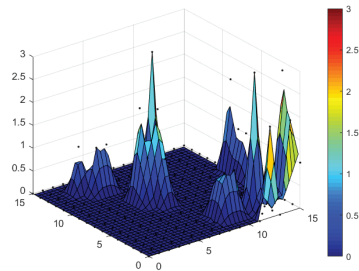

(d)

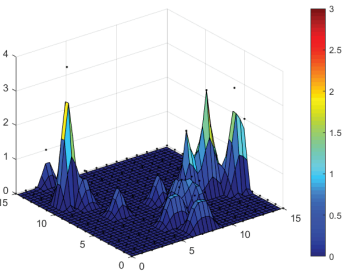

(e)

Fig. 12. $2 \mathrm{D}$ map - neurons activated under healthy conditions, inner race fault, outer race fault, rolling element fault (a) and active areas (b-e). 


\section{Summary}

The presented results show that KNN can be used as a bearing fault detector and classifier with high efficiency. Effectiveness was tested for three types of parameters: map size, distance function and neighbourhood radius. First, results for fault classifiers based on FFT, ENV - data without normalisation, and ENV - normalised data, are compared to emphasise the meaning of input data for KNN. Other results are presented only for ENV - normalised data due to higher efficacy. The most important of the tested parameters is the map size. The larger the map size is, the less important are the other parameters. Based on $8 \times 8$ and $10 \times 10$ maps, it can be noticed that radius equal to 2 and dist function allow procurement of the best results, although more significant discrepancies occur for fault classifier.

In conclusion, KNN can be successfully applied to the diagnostics of the technical condition of rolling bearings provided that normalisation of the input data is applied. ENV analysis is the recommended method for vibration acceleration signal processing. Most of the KNNs tested had detection efficiencies of approximately $98 \%$ and classification efficiency of approximately $93 \%$.

Usually, two accelerometers are used in vibration diagnostics, placed orthogonally on the housing. It is shown that the use of a single accelerometer measuring vibrations in the radial direction allows for high neural detector performance. The proposed approach reduces the cost of the entire investment.

The approach presented in this paper can be used to diagnose the rolling bearings of any rotating machine. The condition to be fulfilled is the selection of appropriate symptoms resulting from the design of the applied bearing (geometrical dimensions of the bearing, number of rolling elements etc.). Based on these dimensions, characteristic symptoms are identified (Eqs (4)-(6)) to be analysed in order to select those that best respond to damage. Proper normalisation of the input data can ensure the universality of the developed detector.

\section{Acknowledgements}

This research was supported by the National Science Centre Poland under grant number 2017/27/B/ST7/00816.

\section{References}

Akar, M., Hekim, M. and Orhan, U. (2015). Mechanical Fault Detection in Permanent Magnet Synchronous Motors Using Equal Width Discretization-Based Probability Distribution and a Neural Network Model. Turkish Journal of Electrical Engineering and Computer Sciences, 23(3), pp. 813-823.

Breard, G. T. (2017). Evaluating Self-Organizing Map Quality Measures as Convergence Criteria. University of Rhode Island: Open Access Master's Theses, Paper 1033.

Ewert, P., Kowalski, C. T. and Orlowska-Kowalska, T. (2020). Low-Cost Monitoring and Diagnosis System for Rolling Bearing Faults of the Induction Motor Based on Neural Network Approach. Electronics, 9(9), pp. 1334.

Ewert, P., Orlowska-Kowalska, T. and Jankowska, K. (2021). Effectiveness Analysis of PMSM Motor Rolling Bearing Fault Detectors Based on Vibration Analysis and Shallow Neural Networks. Energies, 14(3), pp. 712.

Faiz, J., Takbash, A. M. and Mazaheri-Tehrani, E. (2017). A Review of Application of Signal
Processing Techniques for Fault Diagnosis of Induction Motors-Part I. AUT Journal of Electrical Engineering, 49(2), pp. 109-122.

Frosini, L., Harlişca, C. and Szabó, L. (2015). Induction Machine Bearing Fault Detection by Means of Statistical Processing of the Stray Flux Measurement. IEEE Transactions on Industrial Electronics, 62(3), pp. 1846-1854.

Germen E., Başaran M. and Fidan M. (2014). Sound Based Induction Motor Fault Diagnosis Using Kohonen Self-Organizing Map. Mechanical Systems and Signal Processing, 46(1), pp. 45-58.

He, J., Somogyi, C., Strandt, A. and Demerdash, N. A. (2014). Diagnosis of Stator Winding ShortCircuit Faults in an Interior Permanent Magnet Synchronous Machine. In: Proceedings of the 2014 IEEE Energy Conversion Congress and Exposition (ECCE), USA: Pittsburgh, PA.

Henao, H., Capolino, G. A., Fernandez-Cabanas, M., Filippetti, F., Bruzzese, C., Strangas, E., Pusca, R., Estima, J., Riera-Guasp, M. and Hedayati-Kia, S. (2014). Trends in Fault Diagnosis for Electrical 
Machines: A Review of Diagnostic Techniques. IEEE Industrial Electronics Magazine, 8(2), pp. 31-42.

Immovilli, F., Bellini, A., Rubini, R. and Tassoni, C. (2010). Diagnosis of Bearing Faults in Induction Machines by Vibration or Current Signals: A Critical Comparison. IEEE Transactions on Industry Applications. 46(4), pp. 1350-1359.

Jaganathan, B., Venkatesh, S., Bhardwaj, Y. and Prakash, C. A. (2011). Kohonen's Self Organizing Map Method of Estimation of Optimal Parameters of a Permanent Magnet Synchronous Motor drive. In: Proceedings of the India International Conference on Power Electronics 2010 (IICPE2010), New Delhi, India.

Kohonen, T. (2001). Self-Organizing Maps. Berlin, Germany: Springer.

Liu, H., Li, D., Yuan, Y., Zhang, S., Zhao, H. and Deng, W. (2019). Fault Diagnosis for a Bearing Rolling Element Using Improved VMD and HT. Applied Sciences, 9(7), pp. 1439.

Lu, S., He, Q. and Zhao, J. (2018). Bearing Fault Diagnosis of a Permanent Magnet Synchronous Motor via a Fast and Online Order Analysis Method in an Embedded System. Mechanical Systems and Signal Processing, 113, pp. 36-49.

Nkuna, J. S. R. (2006). Vibration Condition Monitoring and Fault Classification of Rolling Element Bearings Utilising Kohonen's Self-organising Maps. Theses and Dissertations (Mechanical Engineering). Ph.D. Thesis, Vaal University of Technology: Vanderbijlpark, South Africa.
Picot, A., Obeid, Z., Régnier, J., Poignant, S., Darnis, O. and Maussion, P. (2014). Statistic-Based Spectral Indicator for Bearing Fault Detection in PermanentMagnet Synchronous Machines Using the Stator Current. Mechanical Systems and Signal Processing, 46(2), pp. 424-441.

Rosero, J., Romeral, L., Rosero, E. and Urresty, J. (2009). Fault Detection in Dynamic Conditions by means of Discrete Wavelet Decomposition for PMSM Running Under Bearing Damage. In: Proceedings of the 2009 Twenty-Fourth Annual IEEE Applied Power Electronics Conference and Exposition, Washington, DC, USA.

Skora, M., Ewert, P. and Kowalski, C. T. (2019). Selected Rolling Bearing Fault Diagnostic Methods in Wheel Embedded Permanent Magnet Brushless Direct Current Motors. Energies, 12(21), pp. 4212.

Skowron, M., Wolkiewicz, M., Orlowska-Kowalska, T. and Kowalski, C. T. (2019). Effectiveness of Selected Neural Network Structures Based on Axial Flux Analysis in Stator and Rotor Winding Incipient Fault Detection of Inverter-fed Induction Motors. Energies, 12(12), pp. 2392.

Ullah, Z., Lodhi, B. A. and Hur, J. (2020). Detection and Identification of Demagnetization and Bearing Faults in PMSM Using Transfer Learning-Based VGG. Energies, 13(15), pp. 3834.

Zhang, J., Wu, J., Hu, B. and Tang, J. (2020). Intelligent Fault Diagnosis of Rolling Bearings Using Variational Mode Decomposition and SelfOrganizing Feature Map. Journal of Vibration and Control, 26(21-22), pp. 1886-1897. 